\title{
Philosophiques
}

\section{La parole du philosophe éthicien est-elle crédible?}

\section{Georges A. Legault}

Volume 17, numéro 1, printemps 1990

URI : https://id.erudit.org/iderudit/027103ar

DOI : https://doi.org/10.7202/027103ar

Aller au sommaire du numéro

Éditeur(s)

Société de philosophie du Québec

ISSN

0316-2923 (imprimé)

1492-1391 (numérique)

Découvrir la revue

Citer cet article

Legault, G. A. (1990). La parole du philosophe éthicien est-elle crédible? Philosophiques, 17(1), 21-43. https://doi.org/10.7202/027103ar

\section{Résumé de l'article}

L'article a pour objectif de poser les accords premiers et nécessaires à l'élaboration de la parole éthique aujourd'hui. Revenant sur la distinction langage - parole, l'A. propose d'orienter la recherche sur la parole (mode dialogique), où le recours aux fondements de la véracité du dire est remplacé par le partage de sens et les raisons de croire. Cette orientation exige de définir le rôle de la philosophie, d'orienter la recherche en éthique sur la délibération humaine et de tenter la corroboration avec des sciences humaines telles la psychologie du développement moral et la psychanalyse. 


\title{
LA PAROLE DU PHILOSOPHE ÉTHICIEN EST-ELLE CREDIBLE ? 1
}

\author{
par Georges A. Legault
}

\begin{abstract}
RÉSUMÉ. L'article a pour objectif de poser les accords premiers et nécessaires à l'élaboration de la parole éthique aujourd'hui. Revenant sur la distinction langage - parole, l'A. propose d'orienter la recherche sur la parole (mode dialogique), où le recours aux fondements de la véracité du dire est remplacé par le partage de sens et les raisons de croire. Cette orientation exige de définir le rôle de la philosophie, d'orienter la recherche en éthique sur la délibération humaine et de tenter la corroboration avec des sciences humaines telles la psychologie du développement moral et la psychanalyse.
\end{abstract}

\begin{abstract}
This article aims to trace the basic agreements necessary to the articulation of ethics today. The $A$. proposes that research be oriented on the dialogical aspect of speech, proposing to insist thus upon reasons to believe and upon the sharing of meaning sharing rather than upon the foundational aspect of ethical language. The orientation supposes that the role of philosophy be redefined, that ethics be centered on human deliberation and that corroborations be searched with other human sciences, namely moral developpernent psychology and psychoanalysis.
\end{abstract}

Nous sommes au printemps de l'étbique ${ }^{2}$. C'est ainsi que Fernand Dumont, en 1986, qualifiait ce moment où notre culture, après le long silence de l'hiver, renoue avec l'éthique. Ce printemps ne signifie pas pour moi que l'éthique était absente de notre

1. Toute question porte déjà son univers de signifiants; c'est pourquoi l'entreprise dia logique commence par l'accord des personnes sur la question instaurant le dialogue. L'objectif du texte se résume à tracer le déplacement théorique imposé par l'accord sur la question posée et la reconnaissance de la pertinence d'un autre point de départ pour une éthique d'aujourd'hui.

2. Dumont, Fernand, «Situation de l'éthique». L'éthique à venir: une question de sagesse? une question d'expertise? Rimouski, Editions du groupe de recherche Ethos, 1987 , p. 20. 
culture, qu'elle n'intervenait pas dans la vie des personnes et des sociétés, ni non plus qu'elle était absente des discours théoriques. Comme la nature n'est pas morte lorsqu'on la croit recouverte d'un linceuil blanc, l'éthique, cette dimension particulière de l'être humain, cherchait, sous l'épais manteau des critiques qui l'avaient réduite au silence, à percer de nouveau. Comme le crocus, cette perce-neige, l'éthique sort du silence pour se manifester par la parole. De privée, elle redevient publique.

L'image du printemps situe bien le climat particulier où la parole éthique d'aujourd'hui essaie de se formuler. Comment un philosophe peut-il parler de l'éthique ? Comment cette parole estelle nouvelle? On ne peut pas oublier que l'on sort de l'hiver. L'ensemble des critiques qui furent formulées contre la morale ne disparaît pas par enchantement parce qu'on préfère utiliser le mot éthique plutôt que celui de morale. Nous devons ainsi faire face aux reproches adressés afin d'évaluer leurs parts de pertinence et de situer l'espace où la parole de l'éthicien peut émerger. La tempête, ne l'oublions pas, s'est manifestée par un constat : Dieu est mort. De cette affirmation, on a conclut culturellement : tout est permis. Cette critique, que certains pouvaient croire définitive, a ébranlé les fondements des morales traditionnelles. Et en l'absence de tels fondements à l'interdit on a conclu à l'absence de validité du discours en éthique. Qu'est-ce qui rend valide le discours de l'éthicien? La réflexion critique nous incite dès lors à cerner, dès les premiers instants de son articulation (section 1), le sens même de l'activité discursive qu'on se propose de faire.

Mais le philosophe éthicien ne peut pas répondre à cette question sans se prononcer sur l'objet même du discours en éthique. Or, s'il est aisé de s'entendre qu'il y est toujours question de bien ou de mal, on ne s'entendra pas aussi facilement pour reconnaître de quoi il est question lorsqu'on en parle. De nouveau, toute spécification apportée à la question «de quoi ça parle en éthique? » (section 2) renvoie à un "pourquoi on en parle? " (section 3). L'objet même de la parole en éthique ne peut se cerner sans une détermination de la fonction du discours éthique dans son adresse à l'autre.

Alors qu'on croyait partir d'une seule question, celle des fondements, nous voilà engagés dans un processus de renvois où le 
philosophe éthicien, invité à interroger le statut de sa parole propose, comme horizon de dialogue, l'éthique dans sa fonction de parole. Le philosophe éthicien perçoit l'éthique comme la parole d'un sujet sur quelque chose adressée à l'autre dans le but de...

\section{LE PHILOSOPHE ÉTHICIEN PARLE}

Tout comme Monsieur Jourdain qui faisait de la prose sans le savoir, le philosophe éthicien parle. Peu importe l'école de pensée, le courant philosophique, les croyances, l'appartenance sociale, il parle. Tout ceci peut paraître un peu risible car ne s'agit-il pas d'une de ces évidences sémantiques? Même à travers le rire, on sait que l'enjeu est ailleurs. Un certain nombre de chercheur-e-s ont pris le virage linguistique pour articuler des modèles d'intelligibilité des phénomènes sociaux. Comme l'indique Julia Kristeva :

Faire du langage, un objet privilégié de réflexion, de science et de philosophie, voilà un geste dont on n'a sans doute pas encore évalué toute la portée. En effet, si le langage est devenu un objet de réflexion spécifique depuis déjà des siècles, la science linguistique elle, est toute récente. Quant à la conception du langage comme «clé » de l'homme et de l'histoire sociale, comme voie d'accès aux lois du fonctionnement de la société, elle constitue peut-être l'une des caractéristiques marquantes de notre époque. ${ }^{3}$

La compréhension des langages et l'analyse de langages premiers que sont les recherches en méta-langages ont été proposées comme modèle de la compréhension. Dans la philosophie anglosaxonne, ces recherches ont constitué en objet d'étude «le discours éthique» ou «le langage de la morale». Par exemple R.H. Hare proposait en 1952 d'étudier la morale par le biais du langage et à partir du modèle du langage prescriptif. ${ }^{4}$ Le discours éthique dans cette orientation philosophique est alors soumis, comme tout autre discours, à l'analyse de sa validité ou bien à celle de ses différentes formulations et usages ${ }^{5}$. Dans la première entreprise,

3. Kristeva, Julia, Le langage cet inconnu. Initiation à la linguistique. Paris, Seuil, p. 9. Voir aussi LegAUlt, G.A., La structure performative du langage juridique. Montréal, P.U.M., 1977.

4. HARE, R.M., The language of morals. N.Y., Oxford University Press, 1964, p. 1.

5. Un exemple de certe conception de l'éthique apparaît dans l'enseignement de l'éthique au niveau collégial dans le cours de philosophie 401 : Éthique et politique. "Qu'est-ce que l'éthique? Voici une définition provisoire : l'éthique est une des sous-disciplines de 
il est objet de critique épistémologique alors que dans l'autre il devient objet de classement et de regroupement.

La philosophie a formulé sa démarche critique en éthique par le biais de son propre modèle du discours et du langage que pose la fonction descriptive ou référentielle comme caractéristique fondamentale. C'est à la lumière de cette structure référentielle qu'on pourra établir la validité d'un discours. On saisit alors toute la portée de la critique épistémologique des discours moraux depuis Hume. En effer, il affirme clairement :

La morale éveille les passions, elle produit ou empêche l'action. La raison est, par elle-même, complètement impuissante sur ce point. Donc les règles de morale ne sont pas des conclusions de notre raison. Personne, je crois, ne niera l'exactitude de cette conséquence ; il n'y a pas d'autres moyens de l'éluder, que de nier le principe qui la fonde. Aussi longtemps qu'on accordera que la raison est sans influence sur nos passions et sur nos actions, il sera vain de prétendre que la morale se découvre seulement par une déduction rationnelle. ${ }^{6}$

Pour lui la question de la validité de la morale se pose en ces termes : que connaissons-nous de l'être pour en déduire ensuite ce que l'humain doit faire en conformité à son être? La critique des fondements de l'éthique s'établit principalement autour de l'absence de la référence du discours philosophique. Dans la mesure où des critères de validité sont reconnus pour tout discours, tous ceux qui ne peuvent s'y inscrire deviennent alors sans signification. C'est la transposition de la position humienne que Carnap effectue ainsi à partir du modèle linguistique propre à notre époque. C'est ce qui lui permet d'affirmer :

La sentence frappe aussi toute philosophie de la valeur ou de la norme ; toute éthique ou esthétique en tant que discipline normative. Si l'on admet en effer une valeur ou une norme (selon les idées de la philosophie de la valeur), on ne peut se référer à aucune expérience de contrôle ; on ne peut pas davantage procéder par déductions à partir de

la philosophie. Elle se préoccupe de l'étude des codes moraux en les comparant les uns aux autres et en tentant d'expliquer pourquoi ils sont si divers, en tentant d'expliquer leur rôle et en tentant de résoudre certains problèmes qu'ils soulèvent. „ BLACKBURN, Pierre, Éthique et politique. Notes de cours, Collège de Sherbrooke, p. 1.

6. Hume, David, Traité de la nature humaine. Tome II. Paris, Aubier Montaigne, 1962, p. 572 . 
propositions expérimentales. Ce que l'on dit n'est donc pas exprimable en énoncés pourvu de sens? 7

La critique épistémologique est radicale parce qu'elle s'insère directement dans la logique des fondements. Si le discours éthique ou moral prétend être fondé sur un savoir ou une connaissance, il doit expliciter la méthode permettant d'atteindre ce statut. L'éthique, comme tout autre discours en science humaine, est soumise au questionnement de sa validité référentielle.

Devant la critique radicale de l'épistémologie, le philosophe éthicien peut réouvrir le débat et consacrer ses énergies à développer une autre conception épistémologique permettant de fonder cette fois le discours éthique tout en respectant les critères de validité reconnus ou en proposer d'autres. Il peut aussi revenir aux discours anciens et prétendre que l'hiver est passé. Mais il peut aussi reconnaître la limite du cadre restreint dans lequel s'enlise le débat épistémologique en éthique. En effet, la critique épistémologique est valide exclusivement pour les personnes qui envisagent le langage comme une structure référentielle, une structure descriptive et non comme une structure intentionnelle. C'est pourquoi il est important de rappeler que le philosophe éthicien parle avant tout. La distinction maintenant classique entre le langage et la parole que nous héritons de Saussure, ${ }^{8}$ s'établit à partir de l'élimination du sujet parlant à un autre sujet dans un acte intentionnel. Il reste alors la structure du langage comme sémantiquement descriptive comme le démontre la critique suivante de Jacques Ellul :

Plus fondamentalement, je me rattacherai à tout le courant de pensée qui fait du langage parlé le spécifique humain. Et ici encore, je lie

7. CARNAP, Rudolf, La science et la métaphysique devant l'analyse logique du langage. Paris, Hermann et Cie, 1934, p. 38. Bien entendu il s'agit pour Carnap de signification cognitive. Les énoncés de valeur ont du sens en tant qu'expression d'émotions. C'est pourquoi l'auteur qualifie dans la même cuvre les philosophes qui s'occupent de ces questions de musiciens sans don musical.

8. En séparant la langue de la parole, on sépare du même coup $1^{\circ}$ ce qui est social de ce qui est individuel $; 2^{\circ}$ ce qui est essentiel de ce qui est accessoire et plus ou moins accidentel. La langue n'est pas une fonction du sujet parlant, elle est le produit que l'individu enregistre passivement; elle ne suppose jamais de préméditation, et la réflexion n'y intervient que pour l'activité de classement... La parole est au contraire un acte individuel de volonté et d'intelligence, dans lequel il convient de distinguer: $1^{\circ}$ les combinaisons par lequelles le sujet parlant utilise le code de la langue en vue d'exprimer sa pensée personnelle $; 2^{\circ}$ le mécanisme psycho-physique qui lui permet d'extérioriser ces combinaisons. SAUSSURE, F. de, Cours de linguistique générale. Paris, Payot, 1967, p. 30 . 
langage et parole; $j$ 'accepte bien entendu que les fourmis aient un «langage» tactile, que les abeilles aient un «langage » visuel, c'està-dire un mode de désignation, de communication, de transmission d'informations à la fois codé et appris. Mais ce « langage » si subtil qu'il soit n'a aucune commune mesure avec le langage parlé de l'homme : il faut être d'abord décidé à ramener le langage à l'information de faits, pour pouvoir identifier ces modes de communication à un langage. ${ }^{9}$

En situant l'éthique comme parole, on refuse certes que la recherche s'enlise dans l'éternel débat épistémologique concernant l'entreprise fondationnelle du langage de la morale. Cependant, il ne s'agit pas d'une politique d'autruche. La question de la validité des discours se transforme en celle de la crédibilité de la parole. La question de la validité des discours comme celle des fondements qui l'accompagne est un monde parmi d'autres pour éviter que la parole s'adresse à des êtres crédules. Puisque la parole de l'éthicien porte sur quelque chose et qu'elle s'adresse à quelqu'un, il faut passer par ce double renvoi si on veut davantage évaluer l'importance de ce déplacement de la validité à la crédibilité.

\section{DE QUOI PARLE LE PHILOSOPHE ÉTHICIEN}

Situer ainsi la question de l'éthique dans le champ de la parole plutôt que dans celui du langage c'est effectivement proposer une autre orientation à la recherche en éthique. Mais si la question de la vérité et des fondements n'est plus à l'ordre du jour, ne reste-t-il pas alors qu'à effectuer le constat de la multiplicité des paroles en éthique et à reconnaître que tout est permis. Ici encore il ne faut pas confondre les conséquences de l'abandon de la recherche des fondements sur une parole en éthique comme affectant toute parole dans le domaine. Face à la question du fondement de l'éthique, on peut distinguer et poser les deux questions suivantes : que recherche-t-on dans l'entreprise de fondation de l'éthique? et quel genre d'éthique est susceptible d'être fondé?

La recherche des fondements de l'éthique vise à éliminer l'arbitraire. C'est une recherche de certitude, un système offrant une garantie aux comportements posés. On peut alors soumettre ce genre d'entreprise intellectuelle à un des deux conseils de Freud

9. Ellul, Jacques, La parole humiliée. Paris, Seuil, 1981, p. 7. Voir aussi, JaCQues, Francis, Dialogiques. Paris, P.U.F., 1979. 
aux philosophes ${ }^{10}$ et s'interroger sur l'arrière-plan psychique de cette entreprise. Le dialogue suivant entre G. Sévérin et F. Dolto illustre bien cette quête de sécurisation sous-jacente à toute entreprise intellectuelle mais aussi le danger inhérent à l'intellectualisation.

- Pour vous (F. Dolto), pas de discussion sur les preuves de l'existence de Dieu, ni sur l'athéisme, ni sur les idéologies.

- Vous savez, pour moi, toutes ces théories sont des systèmes de défense. On veut tout expliquer logiquement. On devient totalitaire par peur que ne se glisse une faille ou une ouverture ou une inconnue dans notre vie.

- Toute théorie devient pour vous, par conséquent, un symptôme?

- Oui, un symptôme est un signe, un indice qui cache et révèle en même temps un état psychique ou une crise. Bien souvent, au lieu d'être un tremplin inconfortable et déséquilibrant pour aller plus loin ou ailleurs, la théorie ou le dogme ou le système de pensée sont des bouche-trous qui colmatent les brèches, gomment les interrogations, endiguent les errances. ${ }^{11}$

Avec l'abandon de la recherche des fondements théoriques d'une morale, la parole éthique s'inscrit alors dans la mouvance même de la vie et de l'expérience humaine, subjective et intersubjective, personnelle et sociale. Ce n'est pas que la théorie devient inutile ou obsolète. Elle quitte alors son lieu impérialiste pour s'adresser comme toute parole à l'échange et au partage comme le souligne la suite de ce dialogue :

- D'après vous, quiconque organise son savoir, agence ses découvertes d'une manière logique, ou dogmatique s'assure contre tout imprévu ou, pire, étouffe ses doutes et fixe ses incertitudes?

- La théorie de quelqu'un ne m'intéresse que si je peux la démonter et la faire mienne, y retrouver, exprimés autrement que je ne l'aurais fait, mes fantasmes, mes découvertes, mon expérience et laisser tomber de la théorie ce qui n'éclaire pas ce que je ressens, ce à quoi je réfléchis, ce que j'expérimente, ce que je vis. ${ }^{12}$

$\mathrm{Au}$ besoin de fonder ne correspond pas n'importe quelle éthique. Avec la critique des fondements on perd le discours éthique qui propose un système moral d'interdictions. Dès qu'on

10. Freud, S., L'intérêt de la psychanalyse, présenté, traduit et commenté par P.L. Assoun. Paris, Recz, 1980.

11. Dolto, F. SÉvérin, G., La foi au risque de la psychanalyse. Paris, Seuil, 1981, p. 10-11.

12. Ibid., p. 11. 
pense la morale comme un système de comportements interdits qui sont traduits par des impératifs, on inscrit le langage moral dans l'horizon du commandement. ${ }^{13}$ De par le type d'acte de discours posé, l'interpellé peut questionner directement si la personne qui interdit possède effectivement l'autorité pour adresser un tel commandement. Le discours de l'interdiction qui ne veut pas être contesté a besoin de poser sa légitimité en montrant les fondements de son autorité. C'est pourquoi la loi morale représente ainsi la loi du père (pour emprunter une image de la psychanalyse et du féminisme) comme loi qui soumet au nom de quelqu'un qui parle. ${ }^{14}$

Diriger la recherche en éthique hors du lieu des fondements c'est alors abandonner la recherche d'un discours d'autorité pouvant légitimement formuler des interdictions afin d'ouvrir un discours du partage de sens de notre agir. La question du bien et du mal qui est au cœur du champéthique n'est plus adressée au comportement en lui-même, la seule composante qui puisse faire l'objet d'une interdiction, mais au sens du comportement, à la raison pour laquelle on pose ce geste plutôt qu'un autre. Renouvelant ainsi avec la question philosophique du pourquoi mais adressée à l'agir humain, le philosophe éthicien demande à l'autre Pourquoi posestu ce geste? En posant ce genre de question, il ne s'agit pas, comme on pourrait le penser, d'une réprimande déguisée où l'on cherche à savoir s'il y a une circonstance atténuante avant de porter un jugement final sur la personne ou son comportement, comme le suppose R. S. Peters dans son analyse de la motivation :

D'abord, nous ne demandons quels ont été les motifs d'un homme que lorsque nous désirons, d'une certaine façon, examiner sa conduite. Le mot est utilisé de façon typique dans les discours moraux ou légaux où les actions doivent être justifiées et non simplement expliquées. Nous attribuons ou imputons des motifs aux autres; nous avouons nos motifs ou nous les gardons pour nous dans nos actions. Ceci explique pourquoi nous demandons souvent quets étaient les motifs lorsqu'il y a

13. L'analyse du langage juridique et la manière dont les philosophes du droit ont posé le problème nous aide à saisir cette tendance à considérer le langage touchant l'agir comme commandement. Sur ce point, G.A. LEGAULt, «Fonctions et structure du langage juridique $»$. Meta, vol. 24, $\mathrm{n}^{\circ} 1$, mars 1979, p. $18-25$.

14. PARENT, P.P., L'expérience éthique et l'expérience du désir, Les cahiers éthicologiques de l'UQAR, $\mathrm{n}^{\mathrm{O}} 14$, janvier 87, PP. $35-46$. 
une brèche dans les attentes conventionnelles; car c'est exactement dans ces sortes de contexte que les hommes doivent justifier leurs actions. ${ }^{15}$

Contrairement à cette approche et plus près de celle exprimée par F. Dolto, la question du pourquoi agis-tu ainsi cherche alors à faire préciser le sens, exprimable en parole, et conforme à la motivation qui nourrit le geste. Ceci permet non seulement la compréhension du geste pour les deux parties en présence, mais surtout le partage du sens. En posant ce genre de question, le philosophe éthicien reconnaît dès lors que l'éthique s'interroge sur la délibération humaine.

La conception que le philosophe éthicien se fait de la parole s'applique de manière circulaire à tout agir humain. L'humain décide et peut orienter sa décision par une délibération portant sur les moyens mais aussi sur les fins. On peut en fait retracer la spécificité des différents courants de pensée en morale à partir de la façon dont ils conçoivent la question de la délibération sur la finalité. ${ }^{16}$

C'est cette capacité humaine de la délibération qui constitue le noyau de la parole en éthique. La délibération a toujours fait partie des morales traditionnelles et occupé une place plus ou moins importante dans les différents systèmes. Le changement d'orientation ne consiste pas à ajouter la délibération mais à la considérer comme ce à partir de quoi la parole en éthique peut s'énoncer. Certains systèmes ont posé les fins dans l'ordre de la nature, d'autres ont posé les fins comme valeurs personnelles ou sociales. Dans ces deux conceptualisations de l'éthique on refuse à l'humain la capacité de délibérer sur les fins. C'est ce que suppose une conception éthique non fondationnelle axée sur la parole. La parole éthique porte ainsi sur la délibération des finalités de l'agir et propose à l'autre, le sens même de l'action, tel qu'il se dégage de la délibération.

Une telle orientation en éthique ouvre la voie à différents niveaux de paroles selon les types de situations où la délibération

15. Peters, R.S., Le concept de motivation. Paris, Les éditions ESF, 1973, p. 32-33.

16. Perelman, Chaim, Olbrechts-Tyteca L., Traité de l'argumentation. La nouvelle rhétorique. Bruxelles, Éditions de l'Institut de Sociologie, 1970, spécialement chapitre 64 : Les fins et les moyens, pp. 368-375. 
est impliquée. Il y a d'abord le niveau personnel, celui du sujet agissant qui est obligé quotidiennement de choisir et d'adopter tel ou tel comportement dans un contexte donné. Évidemment, toute décision n'est pas le fruit d'une délibération. On peut répéter des choix sans pour autant délibérer. Sans entrer dans tous les développements où nous conduirait une théorie de la délibération, on peut au moins convenir que la délibération renvoie à un mouvement de la conscience qui saisit les enjeux conflictuels, pour le sujet agissant, générés par le choix d'un comportement dans le contexte donné, qui porte un jugement de valeur sur eux afin d'établir l'importance relative de chacun des éléments et qui détermine enfin les raisons qui en dernier lieu justifient la priorité accordée à telle valeur dans le contexte donné.

On voit bien ainsi comment la délibération relie dans une unité existentielle, celle de la décision concrète et réfléchie, les éléments de l'axe structural de tout discours moral tel qu'identifiés dans Le Québec face à la formation morale ${ }^{17}$. Ces éléments de l'axe structural sont: la raison, la valeur et le comportement. Cette conception de la rationalité inscrite dans la délibération s'apparente alors à ce qu'Aristote traçait dans l'Éthique à Nico maque comme le type de rationalité de la science politique. Mais il n'y a pas que la délibération personnelle qui est en cause, il y a l'ensemble des délibérations institutionnelles et sociales qui, dans notre système démocratique, nous conduit au juridique et aux assemblées délibérantes.

La parole de l'éthicien est différente lorsqu'elle s'adresse au niveau de la délibération institutionnelle comparativement au niveau individuel, compte tenu du contexte social et démocratique dans lequel elle s'énonce. À moins de prétendre que la délibération personnelle peut se projeter de façon unilatérale sur l'ensemble de la vie sociale, il faut reconnaître les différences de niveaux entre ces deux grandes formes de délibération. Ceci est une conséquence directement impliquée dans le fait de concevoir l'éthique dans le champ de la parole plutôt que dans celui du langage descriptif ou du langage prescriptif. En effer, lorsqu'on conçoit la théorie

17. BEGIN, L., LEGAULT, G.A., Le Québec face à la formation morale. Cahiers de philosophie de l'Université de Sherbrooke. Sherbrooke, Service de la recherche de la Faculté des Lettres et sciences humaines, 1983. 
éthique comme celle qui décrit les devoirs humains tels qu'inscrits dans notre nature, il n'y a pas de différence essentielle entre l'éthique individuelle et l'éthique sociale. Toutes les théories de morale naturelle proposent toujours des modèles où le droit doit être le reflet de cette morale naturelle. ${ }^{18}$ Il suffit de regarder le débat entre les deux mouvements antagonistes pro-vie et prochoix en matière de législation sur l'avortement pour voir à l'œuvre, dans le premier mouvement, l'absence de séparation entre l'éthique individuelle et l'éthique sociale. Mais à partir du moment où cette référence commune à l'universel que représente la Nature n'est plus reconnue, on verra forcément apparaître la différence entre la sphère individuelle et la sphère sociale puisqu'il s'agit de décideurs différents et de problèmes de nature différente. En effet, dans le cas de l'éthique individuelle, nous sommes en présence d'une personne qui, dans une situation conflictuelle, doit entreprendre une action précise. La personne délibère ainsi avec elle-même, dans cette situation particulière, pour choisir un comportement. Dans une telle délibération éthique, la personne est en présence d'elle-même, de ses intérêts mais aussi de cet autre qu'elle peut identifier et qui supportera les conséquences de sa décision. Les questions d'éthique sociale sont d'une autre nature. Les décideurs sont toujours des personnes qu'on peut identifier mais ils occupent une fonction précise qui les place dans une position où l'intérêt de certains individus (quelquefois eux-mêmes) entre en conflit avec l'intérêt de la collectivité (les personnes occupant une fonction élective, de même que les professionnels). Le conflit entre l'intérêt individuel et l'intérêt social caractérise ainsi la problématique de l'éthique sociale alors que le conflit d'intérêt entre deux personnes ou plusieurs personnes marque la particularité de l'éthique individuelle.

Reprenant le débat en matière d'avortement on peut dire que le mouvement pro-choix admet une différence de niveau entre l'éthique individuelle et l'éthique sociale mais on peut s'interroger sur le statut de l'éthique sociale qu'elle véhicule. Certains membres du mouvement pro-choix véhiculent souvent l'idée que la morale est du domaine privé et que le juridique ne doit pas se soumettre à

18. KaliKNOWSKI, G., Le problème de la vérité en morale et en droit. Lyon, Emmanuel Vitte, 1967. 
aucune conception morale. Le juridique doit respecter la liberté de conscience et de religion et limiter les atteintes à la liberté personnelle en mesurant les conséquences des comportements sur la vie physique des autres personnes. Cette conception oppose ainsi la morale au juridique, elle pose deux ordres de normativité comme l'ont développé les philosophies positivistes du droit. ${ }^{19} \mathrm{La}$ normativité juridique est créée par le biais des procédures historiques s'élaborant dans l'espace et le temps. Elle est ainsi un élément des faits sociaux observables. La morale sociale véhiculée par ces membres du mouvement pro-choix est une morale sociale au sens propre du terme, une morale de société, qui traverse le tissu de cette société dans des rapports de force et d'opposition. Dans la mesure où certains membres du mouvement pro-choix s'inspirent de la philosophie positiviste du droit, ils identifient la morale sociale aux mœurs. Une telle conception n'ouvre aucune dimension à la délibération sociale, à une éthique de la délibération puisque les mœurs sont un produit historique.

À partir du moment où l'éthique sociale n'est plus associée à un langage décrivant les devoirs naturels ou à celui du souverain commandant des normes sociales mais à une parole qui s'adresse à un auditoire, on ne peut plus concevoir l'éthique hors du contexte pragmatique de la communication. Le rôle de la parole éthique variera ainsi selon le contexte précis du rapport interpersonnel et du rapport social et dans la mesure des différentes attentes posées dans et par le contexte de communication. C'est ainsi que peuvent s'interpréter les remarques de Ronald Dworkin sur L'impact de la théorie de Rawls sur la pratique et la philosopbie du droit. ${ }^{20} \mathrm{En}$ essayant de répondre à la question, pourquoi la philosophie de Rawls avait eu un impact si immédiat parmi les juristes américains, l'auteur mentionne le malaise des juristes aux prises entre les deux

19. La séparation de la morale et du droit est le thème central développé par la philosophie posiriviste du droit. Les travaux de Hans Kelsen, Alf Ross, H.L.A. Hart s'organisent autour de cette opposition qui est aujourd'hui remise en question. Cf. Georges A. LEGAULT, La structure performative du langage juridique, Première partie, L'évolution des modèles d'intelligibilité du droit dans la philosophie analytique, pp. 35-195. Cetre difficulté d'intégrer un point de vue d'éthique dans le droit est manifeste dans les travaux de la Commission de réforme du droit: V. Les crimes contre le foetus. Document de travail 58, Ottawa, 1989.

20. Dworkin, Ronald, L'impact de la théorie de Rawls sur la pratique et la philosophie du droit. Individu et Justice sociale, autour de Rawls. Paris, Seuil, 1988, PP. 37-53. 
modèles de référence dans la culture anglo-américaine qu'il nomme respectivement : les théories objectives et les théories historiques. Il définit brièvement ces écoles à partir des caractéristiques suivantes :

D'abord, ils pensent qu'il y a des théories de la justice objectives. Ce sont, selon eux, des théories qui se veulent à portée universelle. Elles entendent dire ce que la justice demande dans n'importe quelles circonstances, dans n'importe quelle sociéré, en n'importe quel point de l'histoire. Elles sont rationnelles dans leur méthode, supposant que les principes universels de justice peuvent être déduits par la raison... À ces théories, les juristes opposent ce qu'ils appellent des théories bistoriques de la justice. Ce sont des théories qui ne prétendent pas à l'universalité, mais se présentent seulement comme prévalant dans une société à un moment donné de son histoire. Du point de vue méthodologique, elles sont sociologiques. ${ }^{21}$

C'est dans la mesure où la parole philosophique de J. Rawls présente à son auditoire une perspective permettant d'articuler le rôle de la raison avec les décisions politiques d'une société qu'il rend à nouveau la pensée philosophique disponible à sa société.

Ainsi la parole de l'éthicien à l'un ou l'autre ou aux deux niveaux de délibération, délibération individuelle ou délibération sociale, s'enracine dans une conception de la délibération ellemême et en dernière analyse pose une conception de l'humain. Toute parole en s'adressant à l'autre n'élimine pas pour autant la portée référentielle ou descriptive du langage. C'est pourquoi on peut toujours parler d'un système de représentation immanent à tout discours explicitant au moins les conditions pragmatiques de son articulation. Puisque la délibération en éthique porte sur la finalité de l'agir humain, son explicitation posera nécessairement une conception de l'humain, une vision de l'humain qui, si on arrive à l'articuler, vient préciser le sens ultime de l'agir.

Contrairement au modèle déductif, ce n'est pas la conception métaphysique, empiriste ou déterministe de l'humain qui assure ici la validité de la finalité humaine à partir de laquelle on inscrit la nécessité de telle décision. Non seulement il n'est plus question de la déduction mais la notion de validité perd son sens. L'articulation de la vision de l'humain immanente à la délibération est un

21. Ibid., p. 39-40. 
moment du dialogue entre interlocuteurs où l'un explicite pour lui et pour l'autre, les raisons ultimes de la crédibilité de sa parole dans le contexte individuel, social et culturel qui les unit.

\section{3. À QUI PARLE-T-IL?}

Puisque l'éthicien peut parler à des individus en fonction de leur délibération, à une société (toute assemblée délibérante) ou à toute personne qui propose des modèles d'intelligibilité de l'humain compte tenu de l'objet spécifique de son discours, il ne tient pas avec eux le même discours. La parole de l'éthicien est soumise à une méta-règle de la communication, celle reliant sa parole à son auditoire dans le but de réaliser l'intention même de l'acte de parole. À chacun de ces niveaux, il y a une intentionnalité spécifique qu'il est important de dégager. Sans une connaissance de cette finalité de la communication, il est impossible d'apprécier la pertinence de la parole posée.

Dans la communication inter-subjective, l'éthicien ne vient surtout pas dire quoi faire ou ne pas faire. Il peut agir comme conseiller auprès de certaines personnes qui lui adressent une demande d'aide dans leur délibération. Il ne peut pas s'autoriser à prendre la place de l'autre pour lui dire le bien et le mal mais il peut par contre accompagner l'autre dans cette délibération où les enjeux conflictuels intérieurs empêchent la personne de délibérer. L'art de la question devrait alors servir de point de repère pour permettre à l'autre de regarder l'ensemble de la situation, de sentir le dilemme dans lequel il se situe et de mesurer l'ensemble des conséquences de ses gestes.

Mais le plus souvent, l'éthicien s'adressera à l'autre dans le cadre de l'éducation. Que ce soit dans la vie de tous les jours ou dans une fonction scolaire du primaire à l'université, certaines personnes assumeront la formation de la personne dans sa dimension morale. Cette formation morale soulève ainsi trois questions spécifiques:

- comment l'humain développe-t-il sa capacité de délibération (cette question comprend aussi toutes celles visant à établir ce qui favorise ou empêche ce développement) ?

- comment le discours de l'éducateur peut-il s'inscrire dans cette démarche (cette question comprend toutes celles relatives à la pédagogie de l'enseignement moral)? 
- qu'est-ce que la délibération (cette question renvoie à la théorisation de l'objet central de tous les discours éthiques : la théorie éthique)?

Il ne faut pas oublier que c'est cette question de la formation morale, qu'un jour ou l'autre on se pose pour les siens et sa société, qui a fait émerger la parole éthique et, telle l'hirondelle, a annoncé le printemps de l'éthique. Mais on ne peut pas se poser cette question, sans renouer avec la théorisation de l'éthique. Comment peut-elle alors s'élaborer?

La parole du philosophe éthicien s' inscrit dans l'horizon de la pensée réflexive. Qu'est-ce? Proprement, nous dit le dictionnaire Lalande au mot "réflexion》, retour sur elle-même de la pensée, qui prend pour objet un de ses actes spontanés ou un groupe de ceux-ci. Le philosophe éthicien cherche ainsi à exprimer en parole le mouvement même de la délibération, à partir de son expérience de cette dernière. Cette méthode philosophique dite introspective 22 a fait longtemps l'objet de critiques tant du point de vue de ceux qui partagent les méthodes expérimentales que de ceux dont les recherches s'inscrivent dans l'inconscient. C'est ici qu'est mise en cause la distinction entre la validité du discours et sa crédibilité. Tout discours élaboré à partir d'une méthode réflexive sur l'expérience humaine trace les significations de l'agir humain dans l'ordre de la conscience. Cette parole n'est crédible que par le renvoi à la double expérience, celle dans l'ordre de l'agir et l'autre dans l'ordre de la réflexion et du mouvement de la conscience. Les raisons qui rendent crédibles le discours pourront dès lors s'énoncer et devenir ainsi lieu de dialogue. Il y a une énorme différence dans l'échange lorsqu'il est question de la validité ou lorsqu'il est question de crédibilité. Dans le premier cas l'échange porte sur l'admission du contenu du discours. Rien ne sera admis par les interlocuteurs s'il n'y a pas une preuve démontrant la validité de ce qui est énoncé. Dans l'autre cas, l'échange portera sur le partage du sens de ce qui est dit dans le discours. Même à travers des vocabulaires disciplinaires ou des expressions différentes, deux interlocuteurs pourront partager

22. Introspection: Observation d'une conscience individuelle par elle-même, en vue d'une fin spéculative : $1^{\circ}$ soit en vue de connaître l'esprit individuel en tant qu' individuel; $2^{\circ}$ soit en vue de connaître l'esprit individuel en tant que type immédiatement observable de l'âme humaine en général, ou même de tout esprit, quel qu'il soit. Vocabulaire technique et critique de la philosophie, Lalande. 
le sens de ce qui est dit parce qu'ils partagent déjà les deux expériences à la base dè leur parole. Quant aux personnes qui ne sont pas, comme le dit l'expression courante, sur la même longueur d'onde, ou bien l'échange n'aboutira pas puisque celui qui recherche la validité refusera de partager toute parole n'offrant que sa crédibilité comme garantie, ou bien l'échange deviendra une invitation, une sollicitation à partager, avec beaucoup de méfiance et d'incrédulité, l'univers du crédible.

Ne risque-t-on pas alors de revenir à une autre forme du tout est permis mais cette fois dans l'ordre de la parole? Est-ce que tout humain dès qu'il parle ne s'inscrit pas dans ce double renvoi de l'expérience de base et de celle de la conscience? Alors comment soutenir que tous les discours issus de la méthode réflexive ne sont pas équivalents? Entre différentes conceptions de la délibération, comment est-il possible d'établir une hiérarchie ? En quoi certains discours pourraient-ils être plus crédibles que d'autres?

Puisque le discours philosophique théorique en éthique renvoie à une expérience de base de notre condition d'humain, on devra en conséquence admettre que d'autres recherches entreprises avec d'autres méthodes de connaissance puissent fournir des indications sur le même sujet. Chaque univers disciplinaire apparaît ainsi comme un des discours théoriques crédibles en foncrion des raisons apportées pour sourenir ce discours. Puisqu'aucun ne peut prétendre à la véracité, à être la règle du savoir, les discours théoriques se regrouperont soit par le biais des mêmes règles de crédibilité admises dans la recherche en cause, soit par le biais de la convergence de leurs conclusions. Dans le premier cas, il s'agit d'un accord établi entre la communauté des chercheurs, dans l'autre il s'agit de corroboration entre des recherches disciplinaires différentes. Dans les deux cas, il s'agit de différents moyens pour redéfinir le savoir mais surtout le rôle de la philosophie.

C'est dans le pragmatisme américain tel qu'élaboré par W.v.O. Quine ${ }^{23}$ qu'apparaît clairement le rôle de la communauté scientifique comme élément-clé dans la conceptualisation de la validité du discours scientifique. L'image du discours de la science

23. Begin, L. et al, Pragmatisme et pensée contemporaine. Cahiers de philosophie n ${ }^{\circ} 2$, Sherbrooke, Service de l'édition er de la recherche. F.L.S.H., 1984. 
qu'il évoque souvent est celle du bateau de Neurath. La théorie scientifique est un navire qui doit se refaire sans jamais toucher terre. C'est ce qui le distingue des philosophies qui accordent un statut privilégié aux données sensibles. Il s'oppose à cette école en ces termes:

Ce qui la (son attitude personnelle) distingue peut-être fondamentalement des philosophes de la donnée sensorielle, c'est que j'ai une préférence pour une manière de traiter l'acte de la connaissance qui consiste à le regarder de l'intérieur de la théorie en constante évolution constituant notre connaissance du monde et à refuser d'imaginer qu'un fondement plus solide puisse exister en dehors d'elle. ${ }^{24}$

Le point de départ de la conception de l'auteur c'est la théorie scientifique telle qu'elle se vit dans et par la communauté scientifique. Cette théorie s'appuie sur l'efficacité réelle de ce discours et son amélioration dépend de la maximisation de l'embrigadement qui a servi à la science dans l'élaboration de son langage référentiel. ${ }^{25}$ L'idée de la communauté scientifique brièvement esquissée par Quine par l'énoncé The scientific community is no private club ${ }^{26}$ rejoint en fait celle de Perelman sur l'auditoire d'élite.

Certains auditoires spécialisés sont volontiers assimilés à l'auditoire universel, tel l'auditoire du savant s'adressant à ses pairs. Le savant s'adresse à certains hommes particulièrement compétents et qui admettent les données d'un système bien défini, constitué par la science dans laquelle ils sont spécialisés. Pourtant, cet auditoire si limité est généralement considéré par le savant non comme un auditoire particulier, mais comme étant vraiment l'auditoire universel : il suppose que tous les hommes, avec le même entraînement, la même compétence et la même information, adopteraient les mêmes conclusions. ${ }^{27}$

Comme le mentionnent les auteurs, on peut aussi concevoir la morale selon les mêmes données. La communauté des personnes compétentes dans le domaine pourrait aussi formuler des propositions. Mais il y ane autre façon de voir le discours de l'éthicien. Au lieu d'être un discours fondé sur l'autorité de ses membres ou de ses méthodes, il peut se présenter de façon plus modeste. Au centre de

24. Quine, W.v.O., Le mot et la chose. Paris, Flammarion, 1977, p. 326.

25. Voir particulièrement : Ibid., ch. $\mathbf{~ : ~ L ' e m b r i g a d e m e n t , ~ p . ~ 2 2 7 , ~ e t ~ l e ~ r e j e t ~ d e ~ l ' i n t e n t i o n n a l i t e ́ , ~}$ p. 307.

26. Quine, W.v.O., Ullian, J.S., The Web of Belief. New York, Random house, 1978, p. 3.

27. Perleman, Ch., Olbrechts-TyteCa, L., op. cit., p. 44-45. 
l'interrogation sur le discours de l'éthicien est celle du rôle de la philosophie. Cette question ne peut plus être écartée du débat actuel puisqu'elle est impliquée dans la question de la théorie éthique. Cependant, peu d'écrits interrogent directement sa fonction et ce qu'il reste du discours philosophique alors qu'on conteste ouvertement tous ces penseurs qui assuraient la raison d'être de la philosophie. J. Habermas pose avec clarté le problème et propose La redéfinition $d u$ rôle de la philosopbie. ${ }^{28}$ L'auteur pose le problème de la nécessité de redéfinir le rôle de la philosophie à partir de la critique du fondamentalisme même de la théorie transcendantale de Kant. Selon lui, la conception kantienne propose deux rôles à la philosophie :

... d'une part, une théorie fondamentaliste de la connaissance qui promeut la philosophie dans le rôle de celle qui, vis-à-vis des sciences, définit et indique les places qu'elles doivent occuper et, d'autre part, un système conceptuel anbistorique, plaqué sur la culture dans son ensemble, auquel la philosophie doit le rôle non moins contestable de juge qui règle les juridictions propres de la science, de la morale et de l'art. ${ }^{29}$

Confrontée aux critiques adressées à cette vocation, la philosophie semble s'orienter vers le choix de l'abandon de l'exigence de rationalité qui caractérise son discours. C'est pourquoi Habermas proposera à la philosophie de redéfinir son rôle en coopérant avec les autres entreprises scientifiques. Cette idée de philosophes coopérant avec un esprit faillibiliste, conscients que tout ce dont la philosophie s'était jadis crue capable, dans sa marche solitaire, ne peut désormais plus être espéré que de l'beureuse cobérence de différents fragments théoriques, s'articulera plus précisément dans l'idée de corroboration.

Habermas explique brièvement et applique cette démarche de la corroboration dans Conscience morale et activité communicationnelle. ${ }^{30}$ Partant de son projet de fonder en raison une saisie éthique qui s'appuie sur une théorie de la discussion, l'auteur distingue deux étapes à la fondation. La première qui recoupe celle des sciences reconstructrices en éthique s'inscrit dans la reconstruction des intuitions quotidiennes qui sous-tendent l'évaluation

28. Habermas, J., Morale et communication. Passages, Cerf, 1983, pp. 23-41.

29. Ibid., p. 25.

30. Ibid., pp. 131-204. 
des conflits moraux qui adviennent dans l'action. ${ }^{31}$ Les thèses ainsi formulées ont des prétentions à l'universalité mais leur statut est faible car elles ne peuvent pas être considérées autrement que comme des hypothèses. Cette corroboration s'effectuera directement par le biais des autres théories concurrentes mais il y a une autre exigence. Il faut d'ailleurs ajouter qu'une telle théorie non seulement attend, mais encore exige d'être corroborée indirectement par $d^{d}$ 'autres théories consonantes. ${ }^{32}$ C'est pourquoil'auteur procédera à la corroboration par le biais des travaux du psychologue développementaliste L. Kohlberg ${ }^{33}$ et qui le conduira à s'intéresser aux travaux d'Anna Freud sur les mécanismes de défense.

La délibération humaine ayant été placée comme objet de la parole de l'éthicien philosophe n'est pas l'objet exclusivement de la méthode réflexive en philosophie. La motivation dans l'agir humain est l'objet central de toutes les psychologies qui interviennent au plan thérapeutique. Plus spécifiquement encore, les psychologies développementalistes exploreront selon leur champ d'expérience, le développement de l'intelligence, le développement moral ou le développement de la personne. La psychanalyse, dans ses développements à partir des thérapeutes qui se sont plus particulièrement intéressés à la cause des enfants tels Anna Freud ${ }^{34}$ et Françoise Dolto ${ }^{35}$, s'inscrit aussi dans l'approche développementaliste de la

31. Ibid., p. 131.

32. Ibid., p. 131-132.

33. Kohlberg, L., The Philosophy of Moral Development. San Francisco, Harper and Row, 1981. Gilligan, C. In a Different Voice. Psychological Theory and Women's Development, Cambridge, Harvard University Press, 1982.

34. "Depuis la publication des deux ouvrages de Freud: Psychologie des foules et analyse du moi et $A u$-delà du principe du plaisir qui ont marqué un changement d'orientation, l'anathème de "non-orthodoxe " a cessé de peser sur l'étude du moi et les recherches appliquant aux instances de ce dernier suscitent maintenant un intérêt majeur. Voici comment nous définirons le programme actuel de la psychanalyse qui ne se limite plus à la seule psychologie abyssale. Nous dirons généralement que l'analyse a pour but d'acquérir une connaissance aussi approfondie que possible des trois instances dont l'ensemble constitue, d'après nous, la personnalité psychique, d'étudier leurs rapports mutuels ainsi que leurs relations avec le monde extérieur. » Freud, Anna, Le moi et les mécanismes de défense. Paris, P.U.F., 1949, p. 8.

35. On retrouve la même importance accordée au concept de personnalité dans l'analyse de François Dolto: «Nous allons donner une brève description de la personnalité, mais n'oublions pas qu'il s'agit d'un schéma artificiel et commode pour l'étude, et gardonsnous de voir des compartiments étanches et des entités réelles. On distingue le Ça, le Moi et le Sur-Moi.» Dolto, F., Psychanalyse et pédiatrie. Paris, Seuil, 1971, p. 18 
personnalité. Le philosophe éthicien qui articule une théorie dans le domaine de l'éthique ne peut pas ignorer les autres discours qui se rapportent à la personne humaine, son développement et son agir.

Dans la mesure où il soumettra les conclusions de son discours à l'épreuve de la corroboration, il tentera dès lors de dépasser les limites de la méthode réflexive. Dans le domaine de la recherche en éthique qui tente de préciser le rôle de la raison dans l'agir humain, il y a deux champs disciplinaires que le philosophe éthicien ne peut pas ignorer. Le mouvement de la psychologie développementale s'interrogeant sur le développement moral chez l'humain constitue un secteur de recherche où pourraient s'établir des lieux de corroboration. Ce choix paraît évident compte tenu de la recherche en cause et on saisit pourquoi Habermas s'est intéressé plus particulièrement à ce domaine pour corroborer son entreprise philosophique.

Le second champ par contre risque d'apparaître moins évident, il s'agit de la psychanalyse. Pourtant Freud qualifie lui-même son étude, Malaise dans la civilisation, d'étude sur le bonheur. ${ }^{36}$ Le principe du plaisir tel qu'il s'élabore dans la conception freudienne renvoie ainsi à une formulation psychanalytique des thèmes des discours éthiques axés sur le bonheur. Qu'on prenne par exemple le discours d'Aristote ou celui de J. S. Mill ${ }^{37}$, on retrouve comme référence à la finalité de nos comportements le bonheur et les notions conjointes de plaisir et de peines ou de souffrances.

36. Freud, S., Malaise dans la civilisation. Paris, P.U.F., 1973, p. 32.

37. En effet, cherchant à identifier quel est ce à quoi on tend en toutes circonstances, Aristote le trouve dans la notion de bonheur: Tel parầt être, au premier chef, le bonbeur. Car nous le cherchons toujours pour lui-même, et jamais pour une autre raison. ARISTOTE, Ethique de Nicomaque. Paris, Garnier-Flammarion, 1965, p. 19 et 28. Plus près des notions que l'on retrouve dans Freud, J.S. Mill affirmera : L'utilitarisme soutient au contraire que la seule chose désirable comme fin est le bonbeur, c'est-à-dire le plaisir et l'absence de douleur. J.S. MiLL, L'utilitarisme. Paris, Garnier-Flammarion, 1968 , p. 48. À des conceptions qui situent au centre de l'éthique le bonheur individuel s'opposera celle qui centre ses recherches sur les institutions. Par exemple Simone de Beauvoir précisera sa démarche ainsi : «Nous estimons quant à nous qu'il n'y a d'autre bien public que celui qui assure le bien privé des citoyens; cest du point de vue des chances concrètes données aux individus que nous jugeons les institutions. Mais nous ne confondons pas non plus l'idée d'intérêt privé avec celle de bonheur : c'est là un autre point de vue qu'on rencontre fréquemment; les femmes de harem ne sont-elles pas plus heureuses qu'une électrice? La ménagère n'est-elle pas plus heureuse que 
L'analyse freudienne articule dans son univers théorique la problématique de la souffrance en distinguant aussi trois grandes catégories engendrant la souffrance humaine : la puissance écrasante de la nature, la caducité de notre propre corps, et l'insuffisance des mesures destinées à régler les rapports des bommes entre eux, que ce soit au sein de la famille, de l'État ou de la société ${ }^{38}$. Le malaise de la civilisation provient de cette tension constante entre les forces individualistes et le «sacrifice » imposé par la vie en société. Quel sera le chemin de ces pulsions devant les interdits de la vie sociale? Voilà la question centrale de l'analyse freudienne qui rejoint l'ensemble des discussions en éthique dans une société. L'intérêt de la psychanalyse pour l'éthicien se situe à un premier niveau autour de la théorie des pulsions et leur rôle dans la formation de la personne. En termes psychanalytiques l'émergence d'un sur-moi comme limite au pouvoir des instincts représenté par le Ça, c'est l'émergence d'une conscience morale analysée au niveau de la structuration psychique des personnes dans une société. Cette structuration dans la civilisation se fait avant l'âge de raison et l'émergence du discours conscient. Mais l'intérêt de l'éthicien ne s'arrête pas à ce seul niveau, il concerne aussi l'élaboration même du discours éthique. Il ne faut pas oublier ici la définition freudienne de la civilisation:... la totalité des oeuvres et organisations dont l'institution nous éloigne de l'état animal de nos ancêtres et qui servent à deux fins: la protection de l'bomme contre la nature et la réglementation des relations des bommes entre eux. ${ }^{39}$ Ainsi la parole de l'éthicien (comme celle de toute personne participant à la culture) sera une façon pour un individu déterminé d'assumer le cheminement de ses propres pulsions.

Tant que le discours du philosophe éthicien ne prendra pas le risque de la corroboration, il restera enfermé dans sa méthode réflexive. Mais dans la mesure où ce risque sera assumé, alors la

l'ouvrière? Mais on ne sait trop ce que le mot bonheur signifie et encore moins quelles valeurs authentiques il recouvre; il n'y a aucune possibilité de mesurer le bonheur d'autrui et il est toujours facile de déclarer heureuse la situation qu'on veut lui imposer : ceux qu'on condamne à la stagnation en particulier, on les déclare heureux sous prétexte que le bonheur est immobilité. C'est donc une notion à laquelle nous ne nous référerons pas. " Simone de Beauvolr, Le deuxième sexe. Tome 1. Paris, Gallimard, 1949 , p. 34

38. Freud, S., Malaise de la civilisation, p. 32.

39. Ibid., p. 37. 
corroboration ultime des recherches pourra s'ajouter pour assurer la crédibilité des discours théoriques solitaires.

L'émergence de l'éthique pour aujourd'hui a été au centre de ces propos. L'élaboration d'une éthique pour aujourd'hui ne peut pas faire fi des nombreuses critiques qui lui furent adressées comme produit culturel proposant une réglementation des rapports humains. En fait, le but premier n'était pas de développer en profondeur toutes les thèses présentées mais de cerner ce qui m'apparaît être les accords premiers à établir pour entamer un dialogue fructueux entre les chercheur-e-s dans le domaine de l'éthique. Repris plus scolairement, ces accords pourraient se formuler ainsi :

1. L'éthique d'aujourd'hui doit renoncer à la tentation des fondements et à garantir son discours en lui assurant la validité et s'orienter dans la voie plus modeste de proposer des raisons de croire à la parole énoncée.

2. En prenant la parole plutôt qu'en fondant un langage ou un discours éthique, l'éthicien devrait concentrer sa réflexion sur le lieu de son expérience éthique personnelle et sociale qu'est la délibération.

3. Dans la mesure où il s'interrogera sur la délibération de la personne à qui il parle, sur la délibération institutionnelle ou sur la théorie de la délibération, il n'abordera pas le sujet dans la même perspective. Il devra ainsi reconnaître la spécificité de chacun des niveaux de discours, tant au niveau de l'objet qu'à celui de la fonction discursive.

4. Au niveau théorique, l'objectif sera d'établir, par le biais de sa méthode réflexive, à partir de son expérience humaine personnelle et de celle de la conscience inscrite dans notre héritage culturel, des conclusions sur la délibération humaine.

5. Afin que le discours philosophique de l'éthicien ne demeure pas un discours solitaire, la philosophie devra le soumertre à l'épreuve de la corroboration.

6. Dans cette idée de corroboration de la parole éthique d'aujourd'hui, deux discours ne peuvent être évincés, celui de la psychologie développementale du raisonnement moral et celui de la psychanalyse. 
Il me semble que tout discours qui se propose d'apporter une contribution nouvelle pour actualiser l'éthique en cette fin de $\mathrm{XX}^{\mathrm{e}}$ siècle se doit de partir de ces accords premiers. C'est pourquoi il est important, pour une communauté de chercheur-e-s, de s'arrêter pour discuter des conditions de possibilités de notre dialogue avant de poursuivre respectivement nos travaux.

Département de sciences humaines

Université de Sherbrooke 